\title{
Prime Cancer Vaccine MVA-BN-CV301
}

National Cancer Institute

\section{Source}

National Cancer Institute. Prime Cancer Vaccine MVA-BN-CV301. NCI Thesaurus. Code C143059.

A cancer priming vaccine consisting of a proprietary version of the recombinant vaccinia viral vector, modified vaccinia Ankara-Bavarian Nordic (MVA-BN), encoding both the two human tumor-associated antigens (TAAs) carcinoembryonic antigen (CEA) and mucin-1 (MUC-1), and TRICOM, which is comprised of the three human immune-enhancing costimulatory molecules B7-1, ICAM-1 and LFA-3, with potential immunostimulatory and antineoplastic activities. Upon subcutaneous administration of MVA-BN-CV301, followed by multiple boosting doses of the fowlpox virus (FPV) vaccine CV301, a cytotoxic Tlymphocyte (CTL) response against CEA- and MUC-1-expressing tumor cells is activated. In addition, the CV301-dependent anti-tumor CT L response upregulates the expression of programmed cell death ligand 1 (PD-L1); therefore, when CV301 is combined with a programmed cell death 1 (PD-1) immune checkpoint inhibitor, the antitumor effect may be increased. CEA and MUC-1 are overexpressed in a variety of cancers. 